\title{
The impact of COVID-19 infection of patients with autoimmune blistering diseases of the skin
}

\author{
Ioana-Adriana Popescu, Dumitrița-Lenuța Nichitean, \\ Dan Vâță, Laura Stătescu, Ioana Alina Grăjdeanu, \\ Elena Porumb-Andrese, Laura Gheucă Solovăstru
}

Ioana-Adriana Popescu - M.D., Ph. D. student, Assistant Professor, Department of Dermatology "Grigore T. Popa" University of Medicine and Pharmacy Iași, Romania

Dumitrița-Lenuța Nichitean - M.D., Dermatology Clinic County Emergency Hospital "St. Spiridon" Iași, Romania

Dan Vâță - M.D., Ph. D., Lecturer, Department of Dermatology “Grigore T. Popa” University of Medicine and Pharmacy Iași, Romania

Laura Stătescu - M.D., Ph. D., Lecturer, Department of Dermatology "Grigore T. Popa" University of Medicine and Pharmacy Iași, Romania

Ioana Alina Grăjdeanu - M.D., Ph. D student, Assistant Professor, Department of Dermatology "Grigore T. Popa" University of Medicine and Pharmacy Iași, Romania

Elena Porumb-Andrese - M.D., Ph. D., Assistant Professor, Department of Dermatology “Grigore T. Popa" University of Medicine and Pharmacy Iași, Romania

Laura Gheucă Solovăstru - M.D., Ph. D., Professor, Department of Dermatology “Grigore T. Popa" University of Medicine and Pharmacy Iași, Romania

\begin{abstract}
Nowadays, it is an uncertain time for the whole world, firmly under the grip of a tiny virus, thought to have originated in China. The disease caused by the SARS-CoV-2 virus was termed Coronavirus disease/COVID-19. As dermatologists, we are impacted in multiple ways in the COVID-19 pandemic and have a significant role to play. Autoimmune blistering diseases (AIBD) are a miscellaneous group of disorders associated with autoantibodies directed against distinct desmosomal structural proteins, hemidesmosome structural proteins, or epidermal/tissue transglutaminase. AIBD patients need continuous hospital monitoring, and during this period affected by the pandemic with COVID-19, their physical and mental condition suffered. The cutaneous damage such as bullae formation, pain, itch, and associated
\end{abstract}


functional limitations have a psycho-emotional impact on patients and can severely affect the patient's quality of life.

\section{KEYWORDS:}

Autoimmune bullous diseases, Covid-19 infection, morbidity, quality of life.

\section{INTRODUCTION}

Nowadays, it is an uncertain time for the whole world, firmly under the grip of a tiny virus, thought to have originated in China. A novel enveloped RNA beta corona virus, it was named- Severe acute respiratory syndrome coronavirus due to its structural similarity to the virus causing severe acute respiratory syndrome (SARS). The disease caused by the SARS-CoV-2 virus was termed Coronavirus disease/COVID-19 $(1,2)$.

In December 2019 COVID-19 was reported for the first time, it has spread far and wide across the globe to cause unforeseen health hazards and critical challenges impacting the entire spectrum of life. As doctors, we have a responsibility to support measures which mitigate the spread of severe acute respiratory syndrome coronavirus 2 (SARS-CoV-2), but at the same time, to provide essential medical care to our patients. Cutaneous bullous pathology poses a unique set of challenges ( 1 , 2).

As dermatologists, we are impacted in multiple ways in the COVID-19 pandemic and have a significant role to play. For example, we observe an important psychoemotional impact to patients with autoimmune bullous diseases. Their skin is fragile, the skin barrier has discontinuities and then it would be more at risk to developing Covid 19 infections.

Autoimmune blistering diseases (AIBD) are a miscellaneous group of disorders associated with autoantibodies directed against distinct desmosomal structural proteins, hemidesmosome structural proteins, or epidermal/tissue transglutaminase. These autoantibodies result in the loss of cell adhesion within the epidermis and between the epidermis and dermis, resulting in the cleavage and formation of blisters or erosions.
AIBD occurring in the elderly include bullous pemphigoid (BP), mucous membrane pemphigoid (MMP), epidermolysis bullosa aquisita (EBA), paraneoplastic pemphigus (PNP), pemphigus vulgaris (PV), pemphigus foliaceus (PF), linear IgA dermatosis (LIAD) and dermatitis herpetiformis (DH) (3).

The most common bullous autoimmune dermatosis, bullous pemphigoid is characterized by marked clinical variability and intense pruritus (4). The second most common bullous autoimmune dermatosis, pemphigus vulgaris, typically presents with oral erosions as the predominant and frequently - initial symptom. In Bullous Pemphigoid (BP) subepidermal blistering is induced by autoantibodies against hemidesmosomal structural proteins $(5,6,7)$. Pemphigus vulgaris (PV) is a chronic autoimmune bullous dermatosis that results from the production of autoantibodies against desmogleins 1 and 3 (8).

\section{SKIN CARE IN AIBD}

On the fragility of the skin, it is emphasized that any pressure in apparently normal skin, especially near the injury induces epidermal detachment. Although the individual may present large areas of healthy skin, it is noteworthy that all the skin is vulnerable to further injury (9).

Thus, any pressure on the skin, even during routine nursing care, can increase the injured area, and this a fact confirmed by the positive Nikolski sign (+). This signal is characterized by partial or complete detachment of the epidermis, which is made through the finger pressure on perilesional skin which is found in pemphigus vulgaris. Disruption of bullous lesions gives rise to eroded, exulcerated and exudative lesions, with possible loss of fluid and protein, a group of symptoms that 


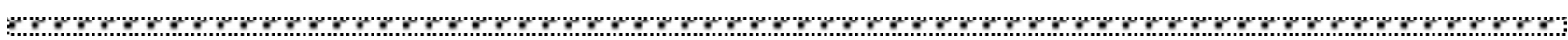

predisposes to infection, dehydration, anemia and deep malnutrition $(9,10)$.

In this period, skin lesions have been reported following frequent hand washing and the use of hand sanitizers. Moreover, skin problems related to personal protective equipment was reported. Aggravation of previous dermatitis has been noted in the wearers and also skin lesions ranging from contact and pressure. Contact dermatitis and hand dermatitis in atopic patients can be a rising concern. The dermatological recommendation is to repeat use of thick emollients and barrier creams to mitigate the damage of the skin.

AIBD patients need continuous hospital monitoring, and during this period affected by the pandemic with COVID-19, their physical and mental condition suffered.

Facing the interference of skin condition in the physical, mental, environmental and social spheres makes the identification of the diagnosis "impaired comfort" understandable. Regarding this diagnosis, there is the pain caused by lack of skin integrity and the difficulty presented by the patients to rest in bed, both interfering negatively in sleep patterns. Moreover, it is necessary to mention the discomfort caused by the patients' exposure to the stigmatizing eyes of society, given the impossibility of hiding a problem that is imprinted on your skin. These factors make the evaluation of that patient in their entirety essential in order to identify the problems and implementation of nursing actions that promote comfort (11).

Always adjuvant to systemic treatment, topical treatment of lesions aims to reduce pain and prevent secondary infection. It is usually performed with corticosteroid and/or antibiotic creams $(12,13)$.

Individual case reports have described a response to topical treatment with the calcineurin inhibitor, tacrolimus. It has mainly been used for localized and limited generalized disease. The use of topical tacrolimus is limited by local irritation and its price compared with topical steroids. It may be useful as an alternative in localized and limited disease without the disadvantage of causing skin atrophy $(14,15)$.

Due to their low immunity, the risk of develop COVID-19 infection increases. In addition, the mandatory wearing of protective equipment favors the appearance of new lesions, with the possibility of bacterial infection.

Blisters should generally be left intact if possible as this may help prevent secondary bacterial infection. When they are particularly large or in sites where they are troublesome or interfere with function, such as the sole of the foot, blisters may be pierced with a sterile needle releasing the fluid, but leaving the blister roof in place, as it forms a natural dressing. If the blister is already broken remove only the fluttering skin (16-18).

If there are extensive areas of erosion and open raw areas, antiseptics such as potassium permanganate as a bath or soaks, or antiseptic-containing bath oils may be used for a few days to dry the lesions and prevent infection (16).

In cases of extensive erosive lesions, the latter may be covered by bandages using different types of dressings, preferably nonadherent, to reduce bacterial superinfection and pain, as well as to promote healing (16-18).

Patients should be monitored for drug sideeffects and to ensure that symptoms are controlled to their satisfaction without excessive doses of topical or systemic treatment. Occasional itching or lesions (if acceptable to the patient) indicates that they are not being overtreated $(16,19)$.

\section{CONCLUSIONS}

It is a difficult period for all of us, especially for patients with autoimmune blistering diseases, due to restricted access to qualified medical care, the obligation to wear protective equipment, with very important implications on psycho-social factors. 
The cutaneous damage such as bullae formation, pain, itch, and associated functional limitations have a psycho-emotional impact on patients and can severely affect the patient's quality of life. These symptoms, by their despicable character, can have a significant burden on social function, independent of clinical severity.

It is important to educate patients and their families about the risks of developing psychiatric side effects during corticosteroid therapy and the need to monitor these patients. Moreover, during this period they must keep in touch with their attending physician to safely continue treatment and to avoid any side effects.

\section{ACKNOWLEDGEMENTS AND DISCLOSURES}

The authors have no potential conflict of interests to disclose.

\section{REFERENCES}

1. Jagadeesan S, Sarkar R. COVID-19 and the dermatologist: finding calm in the chaos. Pigment Int. http://www.pigmentinternational.com/preprintarticle.asp?id=282601

2. WHO Coronavirus disease. https://www.who.int/emergencies/diseases/novel-coronavirus-2019

3. Lakos J. I, Jerkovic G.S, Branka M. Blistering Diseases in the Mature Patient. Clinics in Dermatology. 2017; doi: 10.1016/j.clindermatol.2017.10.014.

4. Hofmann S.C, Juratli H.A., Eming R. 2018. Deutsche Dermatologische Gesellschaft (DDG). Published by John Wiley and Sons Ltd.

5. Mutasim D.F. Autoimmune bullous dermatoses in the elderly: an update on pathophysiology, diagnosis and management. Drugs Aging 2010; 27: 1-19.

6. Sticherling M, Erfurt-Berge C. Autoimmune blisterig diseases of the skin. Autoimmunity Reviews. 2012; 226230.

7. Schmidt E, Zillikens D. Research in practice: diagnosis of subepidermal autoimmune bullous disorders. J Dtsch Dermatol Ges. 2009; 7:296-300.

8. Pollmann R, Schmidt T, Eming R, Hertl M. Pemphigus: a complehensive review in pathogenesis, cinical presentation and novel therapeutic approaches. Clin Rev Allergy Immunol 2018; 54: 1-25.

9. Cunha PR, Barraviera SR. Autoimmune bullous dermatoses. An Bras Dermatol. 2009;84(2):111-24.

10. Brandão ES, Santos I, Lanzillotti RS, Ferreira AM. Et al. Rev. Latino-Am. Enfermagem 2016; 24 : e2766.

11. Santos I, Caldas CP, Gauthier J. at al. Caring for the whole person: the contributions of aesthetics/sociopoetics perspectives to the field of nursing. Rev Enferm UERJ. 2012;20(1):49.

12. Porro AM, Seque CA, Ferreira MCC. At al. Pemphigus vulgaris. An Bras Dermatol. 2019;94(3):264-78.

13. Gach JE, Ilchyshyn A. Beneficial effects of topical tacrolimus on recalcitrant erosions of pemphigus vulgaris. Clin Exp Dermatol. 2004; 29:271-2.

14. Chu J, Bradley M, Marinkovich MP. Topical tacrolimus is a useful adjunctive therapy for bullous pemphigoid. Arch Dermatol 2003; 139:813-15.

15. Calcaterra R, Carducci M, Franco G et al. Topical tacrolimus treatment for localized pretibial bullous pemphigoid. J Eur Acad Dermatol Venereol 2009; 23:177-9.

16. Venning V.A, Taghipour K., Mohd Mustapa M.F.et al. Guidelines for the management of bullous pemphigoid 2012, V.A. Venning et al. British Association of Dermatologists 2012;167:1200-1214.

17. Le Roux-Villet C, Prost-Squarcioni C, Oro S et al. Role of the nurse in care of bullous pemphigoid. Rev Infirm 2010; 160:38-40.

18. Feliciani C, Joly P, Jonkman M.F. et al. Management of bullous pemphigoid: the European Dermatology Forum consensus in collaboration with the European Academy of Dermatology and Venereology. British Journal of Dermatology 2015; 172:867-877.

19. Gheucă Solovăstru L, Vâță D, Stătescu L, Andrese E. - The pshychiatric burden of chronic dermatological diseases. Bulletin of Integrative Pshychiatry. 2014; 3(62): 31-41.

\section{Correspondence:}

Dan Vâță, MD,

"Grigore T. Popa" University of Medicine and Pharmacy, Faculty of Medicine, Dermatology Department, Str. Universitatii nr. 16, 700115, Iasi, Romania, danvata@yahoo.com 